\title{
A Semantic Location Service for Pervasive Grids
}

G. De Pietro - A. Coronato - M. Esposito 


\title{
A Semantic Location Service for Pervasive Grids
}

\author{
G.. De Pietro ${ }^{1}$ - A. Coronato $^{2}-$ M. Esposito ${ }^{1}$
}

\footnotetext{
${ }^{1}$ Istituto di Calcolo e Reti ad Alte Prestazioni, ICAR-CNR, Sede di Napoli, Via P. Castellino 111, 80131 Napoli

${ }^{2}$ Sviluppo ed Applicazione dei Sistemi Informativi Territoriali, SASIT-CNR, Sede di Napoli, Via P. Castellino 111, 80131 Napoli
}

I rapporti tecnici dell'ICAR-CNR sono pubblicati dall'Istituto di Calcolo e Reti ad Alte Prestazioni del Consiglio Nazionale delle Ricerche. Tali rapporti, approntati sotto l'esclusiva responsabilità scientifica degli autori, descrivono attività di ricerca del personale e dei collaboratori dell'ICAR, in alcuni casi in un formato preliminare prima della pubblicazione definitiva in altra sede. 


\title{
A Semantic Location Service for Pervasive Grids
}

\author{
A. Coronato ${ }^{1}$, G. De Pietro ${ }^{2}$ and M. Esposito ${ }^{2}$ \\ ${ }^{1}$ SASIT-CNR, Via Castellino 111, 80131 Napoli, Italy \\ \{coronato.a@na.drr.cnr.it\} \\ ${ }^{2}$ ICAR-CNR, Via Castellino 111, 80131 Napoli, Italy \\ \{giuseppe.depietro,massimo.esposito@na.icar.cnr.it\} \\ $+$
}

\begin{abstract}
In the last decade, Grid Computing and Pervasive Computing paradigms have emerged and rapidly affirmed. These two worlds, however, can no longer be separated islands. As a matter of fact, traditional grids are moving towards Pervasive Grid environments and, as a result, they benefit from both grid and mobile technologies. In particular, a key feature of the Pervasive Computing paradigm, like the location-awareness, can be used to enhance the QoS of existing Grid services, that is Pervasive Grids should customize services access depending on mobile objects locations. But, in these environments, such as in the Pervasive environments, different types of positioning systems can be used to determine mobile objects locations and each of them produces location information with a specific format and granularity. This paper presents a location service that locates active mobile objects, such as Wi-Fi enabled devices and RFID tagged entities, in Pervasive Grids. The key feature of the service is the use of ontologies and rules to define a uniform, unambiguous and well-defined model for the location information, independently from the particular positioning system. Moreover, the location service performs logic and reasoning mechanisms both for providing physical and semantic locations of mobile objects and for giving the location information with the finest granularity when a mobile object is located by more than one positioning system. The service has been developed at the top of the standard OGSA architecture.
\end{abstract}

\section{Introduction}

During the last decade, new computing models have emerged and rapidly affirmed. In particular, terms like Grid Computing and Pervasive Computing have become of common use, not only in the scientific and academic world, but also in business fields.

The Grid Computing paradigm enables resource sharing and coordinated problem solving in dynamic multi-institutional organizations [1]. Grid denotes the 
virtualization of geographically distributed computing and data resources, such as processing, network bandwidth and storage capacity, to create a single system image, granting heterogeneous users and applications seamless access to vast IT capabilities [2].

Differently, the goal for Pervasive Computing is the development of environments where highly heterogeneous hardware and software components can seamlessly and spontaneously interoperate, in order to provide a variety of services to users independently of the specific characteristics of the environment and of the client devices [3]. Therefore, mobile devices should come into the environment in a natural way, as their owner moves, and transparently, that is owner will not have to carry out manual configuration operations for being able to approach the services and the resources.

These two worlds are now evolving towards a common paradigm, namely the Pervasive Grid Computing [4]. As a matter of fact, Pervasive Computing environments can proficiently benefit from grid technologies both to interconnect existing and emerging pervasive environments and to build and deploy new services that require high performance computing and large data resources Errore. L'origine riferimento non è stata trovata..

On the other hand, from the Grid Computing community point of view, it's now time to integrate mobile devices into the grid because they are becoming of common use for accessing to services in any distributed environment.

In particular, a key feature of the Pervasive Computing, like the locationawareness, can be used to enhance the QoS of existing Grid services, that is Pervasive Grid environments should customize services access depending on mobile users and objects locations.

Diverse types of wireless and wired positioning systems can be used to detect presence and proximity of people and mobile objects. The inter-working of more than one positioning system can undeniably provide a synergetic approach of localization, but it requires that Pervasive Grids be supported by advanced location services able to integrate the location information coming from different sources. Indeed, each positioning system produces location data characterized by a specific representation and granularity.

In this paper, we propose a Semantic Location service which localizes mobile objects in Pervasive Grid environments. The service exploits the inter-working of more than one positioning system, by utilizing the Semantic Web technologies [6]. Besides, we define a unique and unambiguous model for localization issues for integrating positioning systems. This model has been applied for integrating two specific types of positioning systems, respectively based on $\mathrm{Wi}-\mathrm{Fi}$ and RFID technologies.

Moreover, the location service performs logic and reasoning mechanisms in order to generate semantic information from physical locations or to give the location information with the finest granularity when a mobile object is located by more than one positioning system.

The service has been developed at the top of the standard OGSA (Open Grid Services Architecture) and so may easily extend traditional OGSA-compliant Grids. 
The rest of the paper is organized as follows. Section 2 discusses some motivations and related work. Section 3 overviews a proposal of location model for a Pervasive Grid. Section 4 describes the location service and outlines the implementation details. In section 5 we present our Pervasive Grid environment and describe some applicative scenarios. Finally, section 6 concludes the paper.

\section{Motivations and related work}

\subsection{Motivations}

Mobile and wireless devices have not been considered, for a long, as useful resources by traditional Grid environments. However, considering the Metcalfe's low, which claims that usefulness of a network-based system proportionally grows with the number of active nodes, and also considering that mobile devices capabilities have substantially be improved over the time, it can justifiably be stated that mobile and wireless devices are now of interest for the Grid community [21].

Over the last years, some valuable efforts have been done in order to make Grid architectures able to support wireless technologies and mobile devices. In particular, the paradigm of Mobile Grid or Wireless Grid has been proposed [5,10,11,12]. More recently, this paradigm has evolved in the Pervasive Grid model [4,5], which again aims at making Grid environments able to integrate mobile devices, but in a pervasive way, that is seamlessly and transparently. In addition to this, the final objective is to enhance Grid environments with characteristics that are typically found in Pervasive environments like the location-awareness.

As a matter of fact, several Pervasive Grid services have to be location-aware, that is they can require location information to cover their needs. But Pervasive Grids, such as classic Pervasive environments, can be equipped with a multitude of different positioning systems able to locate mobile entities.

Ideally, a positioning technology should provide both complete and accuracy location information. In the absence of an ideal solution, multiple technologies are used and each of them can face specific requirements, that is we can utilize a Wi-Fi based system for locating mobile devices or an RFID based system for locating mobile tagged users. Besides, the emerging of new technologies doesn't produce the replacing of the old ones, but the integration of the new and old systems is realized by placing them side by side.

As a result, this increases the complexity of the location-aware services, for the following reasons: i) the services should deal with many and different positioning systems and their low-level protocols; ii) they should know all the specific representations, defined and used by the positioning systems, for location information. Besides, they should convert the location information from each specific representation to an internal one and this produces several different representations for each service; iii) additional location information, such as the semantic location (that is the meaning of a location) should be produced by the specific services. 


\subsection{Our contribution}

This paper proposes an enhanced location service able i) to localize mobile entities by using diverse types of positioning systems and localization techniques and ii) to integrate location information characterized by a specific format and granularity.

In detail, our work consists in the following issues:

- Semantic integration of different positioning systems: The location service exploits the inter-working of more than one positioning system i) by hiding the specific details of the positioning systems and ii) granting both the syntactic as well as the semantic interoperability between services and the positioning systems.

- Definition of a location model: We have defined a location model able to i) provide a unique and uniform representation for location information, independently from the particular positioning system; ii) represent both physical and semantic location of mobile objects of a Pervasive Grid. We have developed the location model by using the Semantic Web technologies and in particular OWL [22] ontologies and SWRL [21] rules.

- Logic and reasoning mechanisms: The location service performs logic and reasoning mechanisms in order to generate semantic information from physical positions or to give the location information with the finest granularity when a mobile object is located by more than one positioning system.

- Integration with the Globus Toolkit: The location service has been developed at the top of the standard OGSA (Open Grid Services Architecture). Then, it has been integrated with the Globus Toolkit [19], which is the the-facto standard platform for Grid applications, and as a result, provides mechanisms for augmenting classic grid environments with a pervasive characteristic like the location-awareness.

\subsection{Related work}

In the last few years location-aware computing and services have been of interest in several research areas, but the mobile devices, and consequently the issues related to the localization, have been largely discriminated by Grid computing. Besides, no relevant experiments have been performed in Pervasive Grids. Differently, a number of pervasive computing systems can be described by the terms location or contextaware and in particular the approaches adopted in [8,9] are illustrated below.

In [8] the author presents a flexible platform for location-based services, which hides specific details of positioning systems and provides a uniform representation of both physical and semantic information. The corresponding infrastructure reflects a location domain model, which defines a semantic structure of the entire location space. This structure is composed of hierarchies that are built up of domains and logical links between domains. A domain represents a semantic location, whereas a link is the expression of a semantic relation between locations. 
Differently, our approach relies on the Semantic Web technologies, and so it grants both the syntactic as well as the semantic interoperability between services and the positioning systems. Besides, the platform proposed in [8] doesn't provide a support for location reasoning, that is no logic mechanisms have been realized for obtaining semantic locations from physical ones or for determining the location information with the finest granularity when a mobile object is located by more than one positioning system.

CoBrA [9] is an architecture to support context-aware services in smart spaces. It locates mobile entities by using two types of positioning systems, respectively based on RFID and Bluetooth technologies. Besides, a set of ontologies has been defined for modelling context information. Semantic Web languages are used for representing them and for supporting context reasoning.

Nevertheless, CoBrA doesn't aim at realizing a semantic integration of different types of positioning technologies. As a result, the context ontologies don't model the location information coming from positioning systems but provide only a uniform and well-defined representation for the semantic locations which can characterize an environment. This choice is purely based on the type of context-aware applications to be supported in prototyping CoBrA.

\section{The Semantic Approach}

\subsection{Our proposal of a location model}

The approach presented in this paper relies on a location model that we have defined to provide a unique and uniform representation for location information, independently from the particular positioning system.

The model is based on the ideas of physical and semantic locations. These notions are not new in literature [13], but we have partially re-elaborated them.

Physical locations specify the position of mobile entities and are characterized by different granularities and scopes, depending on the particular positioning system.

Instead, a semantic location specifies the meaning of a location and usually covers more physical locations. As an example, physical locations can be GPS coordinates, whereas a semantic location can be a building, an office inside a building, a meeting room, a railway station and so on.

In particular, our model describes the physical locations that can be specified by determining the proximity to well-known points, but it can also be easily extended by defining other types of physical locations.

The technique of proximity requires the environment be equipped with infrastructures of sensors, that reveal mobile users presence, or of particular devices to which mobile users can connect, for example to access the Internet. So, the physical locations we have modeled are not referred to coordinate systems, but identify the regions, called sensed areas, covered by a positioning system.

We have focused on two specific types of positioning systems, respectively based on Wi-Fi and RFID technologies, and so we have defined two types of sensed areas: 
a Wi-Fi sensed area, which is identified by the physical location covered by a specific wireless Access Point (AP), i.e. a mobile entity is located by an AP when her mobile device becomes active into its area;

an RFID sensed area, which is identified by the physical location covered by a specific RFID reader, i.e. a mobile entity is located by an RFID reader when her RFID tag is sufficiently near it.

Moreover, we have defined some semantic locations for an environment, as building, room, corridor, and so on. Then, we have subdivided all these semantic locations in many atomic locations, that represent the minimal semantic locations in which a mobile entity can be localized. As an example, an atomic location can contain a desk, a pc, a table or it can be empty.

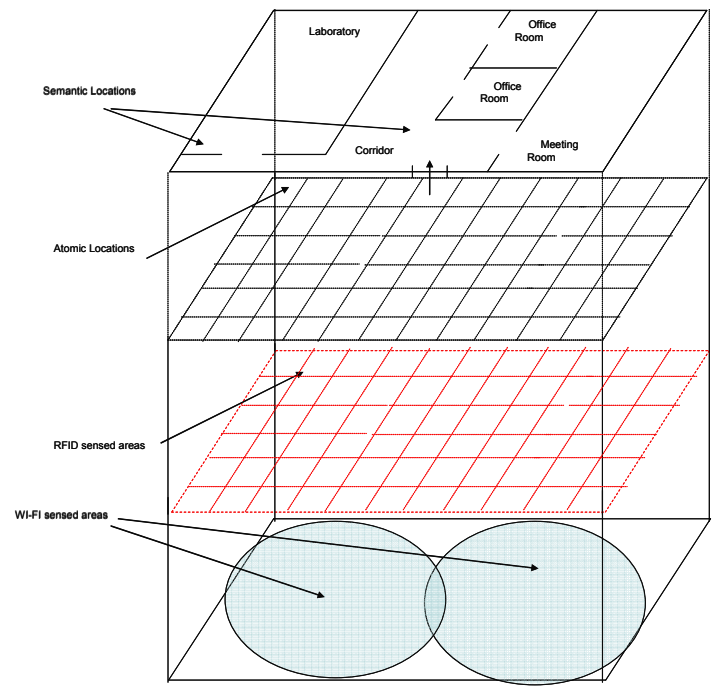

Figure 1 - A representation of our location model

Figure 1 illustrates the relationships existing among semantic locations, atomic locations and sensed areas. Our model is general and, thus, can be applied for describing a large number of location-aware environments. As an example, we can consider the environment in Figure 1, which is composed by semantic locations, such as laboratories, meeting rooms, office rooms, corridors. Each of this location is subdivided in atomic locations (that are identified, in Figure 1, by the rectangular shapes in the black grid).

A sensed area maps one or more atomic location, and, in particular, an RFID sensed area (that is identified by a rectangular shape in the red grid) covers one atomic location, whereas a Wi-Fi sensed area (that is identified by the oval shape) covers more atomic locations. This choice has been motivated by technological issues, that is the RFID based positioning systems we have adopted provide finer grain location information rather than the $\mathrm{Wi}-\mathrm{Fi}$ based ones, even if this is not true in general. As a result, the relationship between a sensed area and one or more atomic 
locations allows to identify the atomic and also the semantic location of a mobile entity.

\subsection{Ontologies and rules for our location model}

The location model defines location information in a unique and uniform way, but these information must be represented in an unambiguous and well-defined formalism and expressed in a machine-readable format. Besides, it has to provide a support for granting both the syntactic and the semantic interoperability between the environments and positioning systems.

The Semantic Web technologies, which have been widely applied in many areas in the recent past, can be used to face such needs because: i) ontologies and rules respectively enable the definition of domain vocabularies and allow declarative data processing, by providing a way to share knowledge without misunderstandings; ii) RDF [10], SWRL and OWL are semantic representation languages with high degree of expressiveness; iii) ontologies and rules can be reasoned by logic inference engines. We can use ontologies and rules coupled with subsets of first order logics to infer new knowledge and to ensure that the system is always in a consistent state.

Therefore, we have modeled three OWL ontologies and a set of SWRL rules related to location issues. In the following, a brief description for the defined ontologies and rules is reported.

The first ontology models all the concepts for defining semantic locations. Location is the root concept of this ontology and represents the generic location of an environment. Each location has a name and can contain or be contained in an other location. Room, Building, Floor and Corridor represent the particular semantic locations of such an environment. They are sub-concepts of the Location concept, and, as a result, each of them inherits the super-concept properties and adds new specialized features to them. For instance, a building is characterized by an address, a number of floors and is composed by corridors, floors and rooms (that is, these concepts are related to the Building concept by a whole-part relationship). We also define different types of rooms, as office-room, meeting-room and laboratory, by specializing the Room concept.

All these possible semantic locations are composed by atomic locations and so all the sub-concepts of Location are related to the Atomic Location concept by a wholepart relationship.

The graphical representation of this ontology is reported in Figure 2.

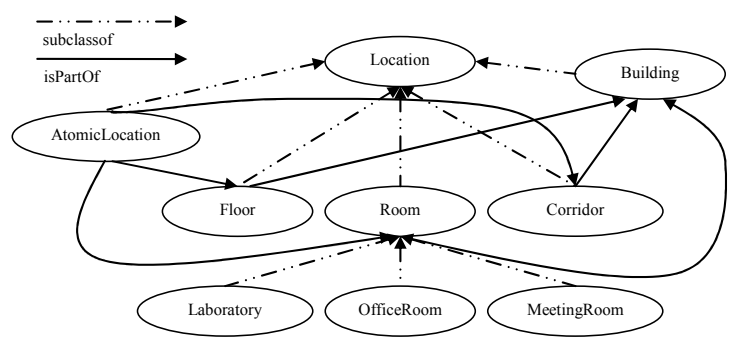


Figure 2 - Ontology for semantic locations

A second OWL ontology identifies the possible entities of an environment. Entity is the root concept and represents the generic entity of the environment. User and Device are sub-concepts of Entity and represent respectively users and devices that can be present in the environment. For instance, a user can be characterized by a name, a surname, an e-mail, a profile and so on.

A positioning system, an RFID Tag and a mobile Pc are particular devices and are described by specific properties. For example, a mobile $\mathrm{Pc}$ is characterized by a set of hardware and software properties, which describe memory capabilities, connection's bandwidth, cpu speed, available applications, and so on.

RFID Reader and Wi-Fi Access Point are sub-concepts of Positioning System and define the two positioning systems we have integrated and described in the model.

A mobile user can be equipped with an RFID Tag or can be the owner of a mobile PC. An RFID Tag can be sensed by an RFID Reader, whereas a mobile PC can be sensed by a Wi-Fi access point.

The graphical representation of this ontology is reported in Figure 3.

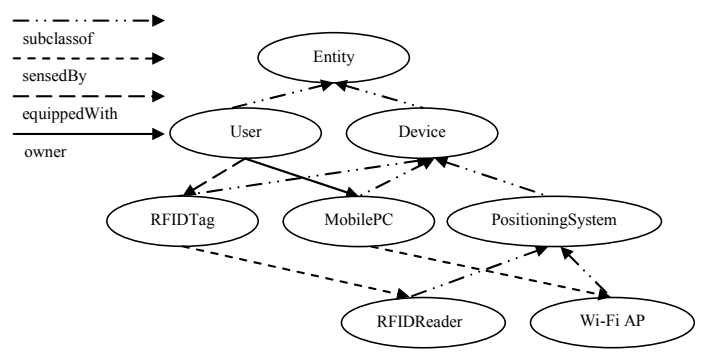

Figure 3 - Ontology for entities of a pervasive grid

The last ontology specifies all the concepts for describing the physical locations. SensedArea is the root concept of this ontology and represents a generic area covered by a positioning system. RFIDSensedArea and Wi-FiSensedArea are sub-concepts of SensedArea and. represent the regions respectively covered by an RFID Reader and Wi-Fi AP. The relationship between a sensed area and one or more atomic locations allows to identify the atomic and, as a result, the semantic location of a mobile user. The graphical representation of this ontology is reported in Figure 4. 


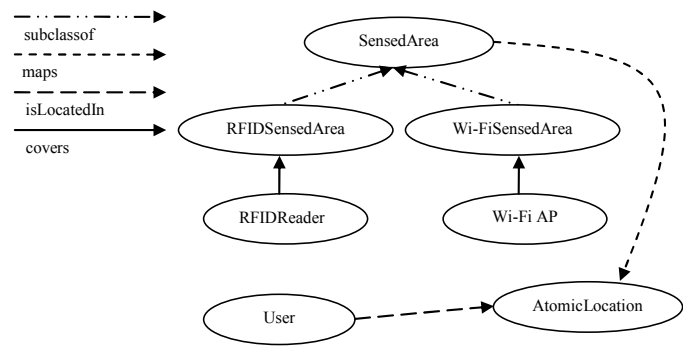

Figure 4 - Ontology for physical locations

Moreover, as an example, we describe two SWRL rules, we have realized on the top of these OWL ontologies, to choice the location information with the finest granularity when an object is located by more positioning systems.

In particular, the next rule makes us able to conclude that, if a mobile entity is sensed by an RFID reader, then it is located in the atomic location associated to the sensed area covered by that RFID reader.

sensedBy(entity, RFID reader) $\Lambda$ covers(RFIDreader, RFIDSensed Area) $\Lambda$ maps(RFIDSensedArea,

AtomicLocation) $\Rightarrow$ isLocatedIn(entity,AtomicLocation)

Instead, by using the second rule, we can conclude that, if a mobile entity is sensed by a Wi-fi AP and is not sensed by an RFID reader, then it is located in the atomic location associated to the sensed area covered by that Wi-Fi AP.

sensedBy(entity, Wi-Fi AP) $\Lambda$ notSensedBy(entity, $R F I D$ reader) $\Lambda$ covers (Wi-Fi AP, Wi-Fi APSensedArea)

$\Lambda$ maps(Wi-Fi APSensed Area, AtomicLocation) $\Rightarrow$ isLocatedIn(entity, AtomicLocation)

If a mobile entity is sensed by both an RFID reader and a Wi-Fi AP, we can obtain the atomic location with the finest granularity by utilizing both the rules shown above. As a matter of fact, there is a condition that is not verified in the second rule (the mobile entity is sensed by an RFID reader), only the first rules hits and thus we can conclude that the mobile entity is located only in the atomic location associated to the sensed area covered by the RFID reader.

\section{The Semantic Location Service}

Current implementation of our location service integrates two distinct positioning systems for locating active mobile objects, like Wi-Fi enabled devices and RFID tagged entities. It has been developed in a real Pervasive Grid environment.

It provides both location and locating functions. A location function is a mechanism for identifying objects active at a specific physical location, whereas a locating function is a mechanism for identifying the location of specific objects [16].

The service architecture consists of the following components: 


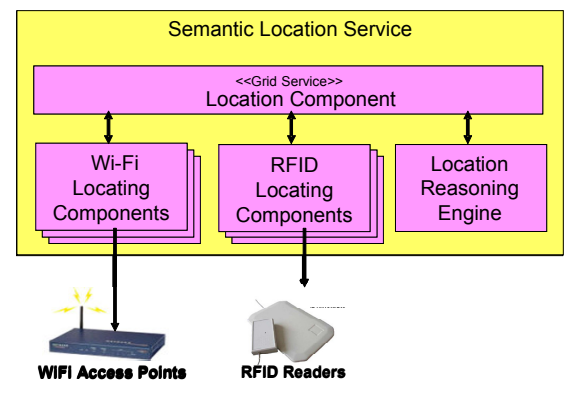

Figure 5 - The Location service architecture

The location service has been realized as an OGSA-compliant Grid Service. It has been developed and integrated in the Globus Toolkit [20], extending the open source collection of OGSA-based Grid Services offered by it.

Currently, we have developed two locating components that identify the location of mobile objects by using respectively the Wi-Fi and RFID based positioning systems. New locating components for other positioning technologies, such as the Bluetooth technology, can be realized and easily integrated in the service.

The Wi-Fi Locating Component is in charge of locating Wi-Fi enabled mobile devices, by periodically interrogating Wi-Fi APs. Indeed, each AP writes an event into a log file whenever a device becomes active into its area. By comparing such logs and by handling global states, it is possible to detect location changes. A similar approach has been realized in [17]. This technique can not be used to recognize when a device becomes inactive, because hand-offs are not reported in the log file. It is possible to adopt a strategy based on checkpoints, and in particular the environment can periodically detect each mobile device with a ping operation. After having issued a ping message, the environment waits for a response or for a timeout. A mobile device is declared inactive after having missed a certain number of consecutive ping messages. Current implementation uses 3Com Office Connect Wireless 11g Access Points.

The RFID Locating Component is in charge of locating RFID tagged entities, by periodically interrogating RFID readers. When an entity is sensed by a reader, we can obviously conclude that it is located in the atomic location covered by that reader. A similar approach has been described in [18]. Current implementation uses the passive, short-range (30 cm), Feig Electronic RFID, model ISC.MR 100/101.

The Location Component is in charge of handling global location states obtained by combining information coming from Locating components.

The Location Reasoning Engine is the core-component of the location service and is in charge of managing the location ontologies and rules. First of all, it makes use of the location ontologies and rules to specify location information in a uniform representation. As a result, it is able to grant both the syntactic as well as the semantic interoperability between the environment and any positioning system.

Besides, it uses a logic inference engine to perform reasoning mechanisms about the location information. In particular, our location ontologies and rules are submitted to the inference engine and reasoned by it in order to i) generate the semantic 
locations of mobile entities from their physical locations and ii) give the location information with the finest granularity when a mobile entity is located by more than one positioning system.

Nevertheless, the Location Reasoning Engine cannot use SWRL rules and OWL ontologies in a unique inference engine, because a complete integration of them in a unique system is inapplicable because of decidability issues. Besides, a solution based on a stack of inference engines doesn't represent an efficient, simple, and scalable solution for obtaining a sound and complete reasoning process.

The solution we have adopted is based on i) the use of a unique inference engine for rules and ii) the translation of OWL ontologies in SWRL rules. But, OWL syntax constructs can not all be translated into SWRL rules, and so we have used the DLP OWL language [19]. It represents the OWL subset that can be translated in SWRL, that is DLP OWL ontologies can be translated into SWRL rules and vice versa.

So the Location Reasoning Engine translates DLP OWL ontologies into SWRL rules and then it is able to use a unique rule engine to infer and reason in a complete and sound way. It is worth noting that DLP OWL is less expressive than either the ontology or rule languages, but, in many cases, the complete expressiveness of OWL is not needed and a restriction such as DLP OWL is enough.

\section{An experimental scenario}

Grid applications can be physically placed in different sites, which are topologically organized to compose intra-Grids, extra-Grids or inter-Grids [14,15].

The experimental scenario consists of an intra-Grid, composed by diverse physical site located in a three floors building. The virtual environment uses two floors of the building.

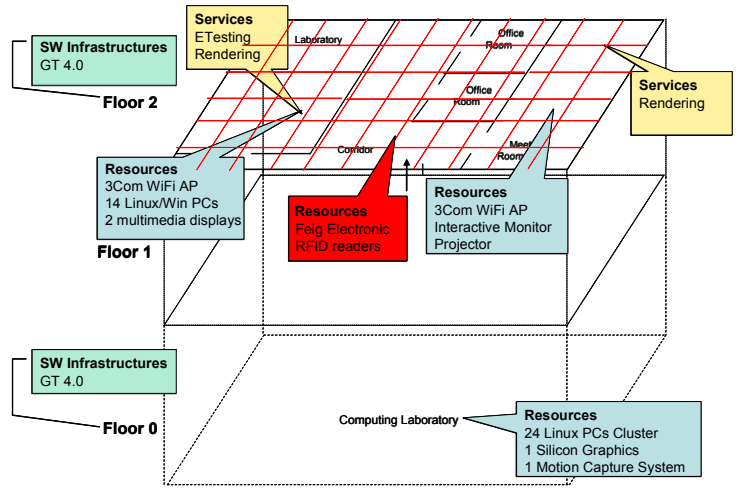

Figure 6 - Pervasive Grid Architecture

Floor zero has a computing laboratory in which a cluster of 24 linux PCs, a 12 processors Silicon Graphics workstation, and a motion capture system are deployed. Such resources are collected in a wired grid built at the top of the Globus Toolkit 4.0 platform. 
On floor two, wireless access to the grid is available. As a matter of fact, two 3Com Office Connect Wireless 11g Access Points identify two distinct locations. L1 is a laboratory where our students develop their activities and periodically perform ETests. It contains two multimedia displays and some desktop PCs. L2 is a meeting room equipped with a projector, an interactive monitor, and other multimedia devices. The floor two is subdivided in atomic locations, and each of them is equipped with a Feig Electronic RFID reader.

Some services are available:

- RenderingService - This service enables users to submit row motion data and to build 3D graphic applications. This service is exposed as a Grid Service and is available at every location (L1, L2);

- ETestingService - This service performs on-line evaluation tests for courseware activities. Evaluation tests are synchronized and students have a predefined period of time for completing each test section. This service is exposed as a Grid Service, but it must be available only in the laboratory (L1).

In the last part of this section, we present some example scenarios in which the presence of a location service is required for supporting Pervasive Grid environments. Specifically, we refer to this Pervasive Grid architecture and describe our services that need information about mobile entities position.

A first possible scenario can be represented by an evaluation test which can be performed in the laboratory of our Pervasive Grid. The access to this service has to be enabled only to the students which are physically located in the multimedia laboratory in which the test is executed. When a student, equipped with and RFID tag, enters the laboratory and stays at a specific atomic location, the RFID reader placed in it reveals his presence and loads test on his desktop pc. If the student leaves the atomic location during the test execution, the RFID reader reveals his movement and, as a result, the test is interrupted and any resources associated to it have to be released.

Another possible scenario is a mobile user which launches a rendering operation submitting it to the rendering service to build 3D graphic applications. When the results are ready, this service returns them to the user depending on his position, that is:

- if a WI-FI AP locates the user, for example, in the laboratory, the results can be indiscriminately presented on one of the displays placed in it, rather than on his mobile device;

- if the user is also equipped with an RFID tag and his presence in the laboratory is revealed by both a WI-FI AP and an RFID reader, the location information with the finest granularity, that is the one produced by the RFID reader, is used and, as a result, the rendering service presents the results on the display placed in the atomic location nearest the user.

\section{Conclusions and future work}

In this paper we presented a Semantic Location service locates active mobile objects, such as Wi-Fi enabled devices and RFID tagged entities, in Pervasive Grids. The key feature of the service is the use of ontologies and rules i) to define a uniform, unambiguous and well-defined model for the location information, independently 
from the particular positioning system; ii) to perform logic and reasoning mechanisms for providing physical and semantic locations of mobile objects and for giving the location information with the finest granularity when a mobile object is located by more than one positioning system. This facility provides the Pervasive Grid with support for customizing services depending on the user location, as well as enabling mobile users to get access.

Future work will aim to realize locating component for integrating new positioning technologies, such as Bluetooth. As a result, the location model will be extended by defining the concepts related to these new positioning systems, and in particular the new types of physical locations provided by them.

\section{References}

[1] I.Foster, C. Kesselman, S. Tuecke, "The Anatomy of the Grid: Enabling Scalable Virtual Organizations", International J. Supercomputer Applications, 2001

[2] "IBM Grid Computing" web site, http://www-1.ibm.com/grid/about_grid/what_is.shtml.

[3] D. Saha and A. Murkrjee, "Pervasive Computing: A Paradigm for the 21 st Century", IEEE Computer, March 2003.

[4] V. Hingne, A. Joshi, T. Finin, H. Kargupta, E. Houstis, "Towards a Pervasive Grid", International Parallel and Distributed Processing Symposium, IPDPS 2003.

[5] N. Daves, A. Friday, and O. Storz, "Exploring the Grid's Potential for Ubiquitous Computing", IEEE Pervasive Computing, April-June 2004.

[6] "Semantic Web" web site, http://www.w3.org/2001/sw/.

[7] L. W. McKnight, J. Howinson, S. Bradner, "Wireless Grids", IEEE Internet Computing, July-August 2004.

[8] J.Roth, "Flexible Positioning for Location-based Services", IADIS Journal on WWW/Internet, Vol. I, Nr. 2, Dez. 2003, IADIS Press, 18-32

[9] Harry Chen, Filip Perich, Dipanjan Chakraborty, Tim Finin, Anupam Joshi, "Intelligent Agents Meet Semantic Web in a Smart Meeting Room", Department of Computer Science \& Electrical Engineering, University of Maryland, Baltimore County

[10] B. Clarke and M. Humphrey, "Beyond the 'Device as Portal': Meeting the Requirements of Wireless and Mobile Devices in the Legion of Grid Computing System", International Parallel and Distributed Processing Symposium, IPDPS 2002.

[11] T. Phan, L. Huang and C. Dulan, "Challenge: Integrating Mobile Devices Into the Computational Grid", International Conference on Mobile Computing and Networking, MobiCom 2002.

[12] D. C. Chu and M. Humphrey, "Mobile OGSI.NET: Grid Computing on Mobile Devices", International Workshop on Grid Computing, GRID 2004.

[13] S.Pradhan, "Semantic locations", Personal Technologies, Vol. 4, No 4, 2000, 213-216.

[14] L. Ferreira, V. Berstis, J. Armstrong, M. Kendzierski, A. Neukoetter, M. Takagi, R. BingWo, A. Amir, R. Murakawa, O. Hernandez, J. Magowan, N. Bieberstein, "Introduction to Grid Computing with Globus", IBM RedBooks, September, 2003.

[15] J. Joseph, M. Ernest, C. Fellenstein, "Evolution of grid computing architecture and grid adoption models", IBM Systems Journal, December, 2004.

[16] S. Fischmeister, G. Menkhaus and A. Stumpfl, "Location-Detection Strategies in Pervasive Computing Environments", in the proc. of the 1st international conference on Pervasive Computing, PERCOM03.

[17] S. G. M. Koo, C. Rosenberg, H. H. Chan, Y. C. Lee, A. Vilavaar, A. Wenzel, "LocationBased E-Campus Web Services: From Design to Deployment", in the proc. of The first 
IEEE International Conference on Pervasive Computing and Communications, PERCOM 2003.

[18] J. Hightower and G. Borriello, "Location Systems for Ubiquitous Computing", IEEE Computer, August 2001.

[19] Grosof B., Horrocks I., Volz R., and Decker S., "Description Logic Programs: Combining Logic Programs with Description Logic", In: Proc. 12th Intl. Conf. on the World Wide Web (WWW-2003), Budapest, Hungary, May 20-23, 2003.

[20] I.Foster, C. Kesselman, J. Nick, S. Tuecke, "The Physiology of the Grid: An Open Grid Services Architecture for Distributed Systems Integration”, Open Grid Service Infrastructure WG, Global Grid Forum, June 22, 2002.

[21] Ian Horrocks, Peter F. Patel-Schneider, Harold Boley, Said Tabet, Benjamin Grosof, Mike Dean, "SWRL: A Semantic Web Rule Language Combining OWL and RuleML", W3C Member Submission 21 May 2004. Latest version is available at http://www.w3.org/Submission/2004/SWRL/.

[22] Peter F. Patel-Schneider, Pat Hayes and Ian Horrocks, "OWL Web Ontology Language Semantics and Abstract Syntax", W3C Recommendation 10 February 2004. Latest version is available at http://www.w3.org/TR/owl-semantics/.

[23] Graham Klyne, Jeremy J. Carroll, and Brian McBride, "Resource Description Framework (RDF) Concepts and Abstract Syntax.", W3C Recommendation 10 February 2004. Latest version is available at http://www.w3.org/TR/rdf-concepts/. 